\title{
Función tiroidea en escolares con obesidad.
}

\author{
Nerkis Angulo ${ }^{1}$, Sobeida Barbella de Szarvas ${ }^{2,3}$, Dora Gonæáles ${ }^{1}$, Ana Hernándes ${ }^{1}$ \\ y Anairis Escobar ${ }^{4}$ \\ ${ }^{1}$ Departamento de Ciencias Morfológicas y Forenses de la Escuela de Ciencias \\ Biomédicas y Tecnológicas, Universidad de Carabobo, Valencia, Venezuela. \\ ${ }^{2}$ Unidad de Investigación en Gastroenterología y Nutrición Pediátrica de la Universidad \\ de Carabobo, Valencia, Venezuela. \\ ${ }^{3}$ Departamento Clínico Integral del Sur. Valencia, Venezuela. \\ ${ }^{4}$ Departamento de Investigación y Desarrollo Social. Escuela de Bioanálisis, Universidad \\ de Carabobo, Valencia, Venezuela.
}

Palabras clave: obesidad en escolares; tiroides; antropometría; insulina.

Resumen. La obesidad se acompaña con frecuencia de trastornos metabólicos, como la resistencia a la insulina, dislipidemias, hipertensión arterial, diabetes mellitus tipo 2 y modificaciones en los niveles plasmáticos de determinadas hormonas. Entre las alteraciones hormonales, la disfunción tiroidea es la más común. El hipotiroidismo puede favorecer la obesidad, pero múltiples teorías apuntan a que es la obesidad, la que genera disfunción tiroidea. El objetivo de la investigación, fue determinar los niveles de hormonas tiroideas en escolares con obesidad y analizar su relación con las variables antropométricas, niveles séricos de insulina y el índice HOMA1-IR. Este fue un estudio observacional, analítico y prospectivo, con un diseño transversal de serie de casos, realizado en 90 niños entre 7 y 12 años de edad, en el servicio de Gastroenterología y Nutrición Pediátrica de la Ciudad Hospitalaria "Dr. Enrique Tejera", de Valencia, Venezuela. Se analizaron los valores de TSH, T3 y T4 libre, y se correlacionaron con sexo, condición socioeconómica, maduración sexual, índice de masa corporal, área grasa del brazo, circunferencia de cintura, insulina basal e índice HOMA1-IR. El 25,44\% resultó hipotiroideo y el resto eutiroideo. En los escolares con obesidad y T4 libre baja hubo una correlación significativa $(\mathrm{p}<0,05)$ con hiperinsulinismo y presentaron promedios significativamente mayores $(\mathrm{p}<0,05)$ de HOMA1-IR. En conclusión, se encontró una frecuencia elevada de hipotiroidismo en escolares con obesidad.

\footnotetext{
Autor de correspondencia: Nerkis Angulo, Departamento de Ciencias Morfológicas y Forenses de la Escuela de Ciencias Biomédicas y Tecnológiicas, Universidad de Carabobo, Valencia, Venezuela. Tlf. 58-0412-5005476. Correo electrónico: nerkis.ang@gmail.com
} 


\title{
Thyroid function in obese schoolchildren.
}

\section{Invest Clin 2021; 62 (1): 5-15}

Key words: obesity in schoolchildren; thyroid; anthropometry; insulin.

\begin{abstract}
Obesity is often accompanied by metabolic disorders, such as insulin resistance, dyslipidemias, high blood pressure, type 2 diabetes mellitus and modifications in plasma levels of certain hormones. Among the hormonal disorders, thyroid dysfunction is the most common. Hypothyroidism ean promote obesity, but multiple theories suggest that it is obesity, which causes thyroid dysfunction. The objective of the research was to determine thyroid hormone levels in obese schoolchildren and analyze their relationship to anthropometric variables, serum insulin levels and the HOMA1-IR index. This was an observational, analytical, prospective study with a cross-sectional design of case series, conducted in 90 children between 7 and 12 years of age, at the Gastroenterology and Pediatric Nutrition Services of the Hospital City "Dr. Enrique Tejera", Valencia, Venezuela. TSH, T3 and T4 free values were analyzed and correlated with sex, sexual maturation, body mass index, arm fat area, waist circumference, basal insulin and HOMA1-IR index. Of the subject studied, 25.44\% were hypothyroid and the rest euthyroid. In schoolchildren with obesity and low free $\mathrm{T} 4$, there was a significant correlation $(\mathrm{p}<0.05)$ with hyperinsulinism and they had significantly higher averages $(\mathrm{p}<0.05)$ of HOMA1-IR. In conclusion, a high frequency of hypothyroidism was found in obese schoolchildren.
\end{abstract}

Received: 25-05-2020 Accepted: 08-12-2020

\section{INTRODUCCIÓN}

Actualmente, muchos países de ingresos medios y bajos están afrontando una «doble carga» de morbilidad; mientras continúan encarando los problemas de las enfermedades infecciosas y la desnutrición, también experimentan un aumento en la prevalencia de la obesidad, en contraste con algunos países de ingresos altos, donde la obesidad se ha mantenido e incluso ha disminuido (1). En el último informe publicado por la Organización Mundial de la Salud (OMS), en el 2019 con los datos correspondientes al año 2018, la prevalencia de sobrepeso y obesidad fue de 5,9\%, que representan 40,1 millones de niños en todo el mundo, lo que pone en evidencia que es un problema que reviste atención y que debe manejarse de forma inmediata (2).

En la mayoría de los niños la obesidad es de origen primario, producida por un desbalance entre la energía que ingresa a través de los nutrientes y el gasto energético; generalmente se acompaña de modificaciones en los niveles plasmáticos de determinadas hormonas con cambios en sus patrones de secreción y/o aclaramiento (3). Entre las alteraciones hormonales, la disfunción tiroidea es la más común (4). Si bien el hipotiroidismo puede favorecer la obesidad, múltiples teorías apuntan a que es la obesidad en sí la que genera disfunción tiroidea (5). Aunque no están claros los mecanismos fi- 
siopatológicos, la alteración más evidente es el aumento de TSH (hormona tiroestimulante de la tiroides), por lo que se ha propuesto, que en sujetos con obesidad, este aumento forma parte de un proceso de adaptación al aumento del metabolismo energético basal. Existiendo una relación entre los trastornos hormonales de la tiroides y los cambios metabólicos relacionados con la obesidad (hipertensión, dislipidemia, hiperǵlucemia, resistencia a la insulina, enfermedad del hígado graso no alcohólico) en niños con obesidad (6). Es necesario considerar que los niveles de las hormonas tiroideas varían según la técnica de medición utilizada, la edad, el desarrollo puberal, el índice de masa corporal (IMC), la obesidad central y los valores de leptina e insulina plasmáticas $(7,8)$.

La prevalencia internacional de hipotiroidismo subclínico en niños obesos, ha sido reportada entre 7,5 y $23 \%(6,7)$; en escolares prepúberes obesos Mexicanos, fue de $11,3 \%$ (9) y en la India de 30,2\% (10). En Valencia,Venezuela para el año 2017 reportaron en niños y adolescentes con sobrepeso, una prevalencia de 6,3\% (11).

El diagnóstico de las alteraciones del eje hipotálamo-hipófiso-tiroideo, se basa en el reconocimiento de una disfunción de dicho eje y en la identificación de su etiología (12). La introducción de métodos sensibles para medir la TSH y las hormonas tiroideas libres: T4 libre (tiroxina) y T3 libre (triyodotironina), ha simplificado mucho el reconocimiento de las disfunciones tiroideas, entendiendo por tales la alteración de la concentración sérica de cualquiera de ellas (13).

Evaluar la asociación entre obesidad y disfunción tiroidea, es de interés porque las hormonas tiroideas afectan la regulación del metabolismo energético y los escolares son el grupo etario que más consulta por obesidad. El objetivo del estudio fue, analizar la relación de los niveles de hormonas tiroideas en escolares con obesidad y las variables antropométricas y la insulina.

\section{PACIENTES Y MÉTODOS}

Estudio observacional analítico, prospectivo, con un diseño transversal, de serie de casos (obesidad). La selección de la muestra fue de tipo no probabilística, intencional, es decir todos los niños que cumplieran los siguientes criterios:

- Edad entre 7 y 12 años; escolares que acudieron a la consulta de nutrición en el servicio de Gastroenterología y Nutrición Pediátrica de la Ciudad Hospitalaria "Dr. Enrique Tejera", de Valencia. Se usó la edad escolar porque es el grupo etario, que consultó con mayor frecuencia por sobrepeso y obesidad.

- IMC > p97, siguiendo el patrón de referencia de la OMS (2007) (14).

- Consentimiento informado: los padres, tutores o representantes legales, tras haber recibido la información adecuada en forma verbal y escrita, proporcionaron previamente su consentimiento informado y los niños mayores dieron su asentimiento. El protocolo de estudio fue aprobado por el Comité de Docencia e Investigación y el Comité de Ética en Investigación de la Ciudad Hospitalaria "Dr. Enrique Tejera" de la ciudad de Valencia,Venezuela.

- Obesidad exógena y maduración sexual Tanner I (prepúberes) y Tanner II: determinado por las características de glándula mamaria, vello axilar y pubiano en las niñas, y genitales, vello axilar y pubiano en los varones (15).

- Criterios de exclusión: presencia de enfermedades crónicas y consumo de fármacos que pudieran alterar la función tiroidea (anticonvulsivantes, litio) o corticoides, puesto que su uso en dosis altas y de forma prolongada, en el tratamiento de diversas patologías, puede originar un Síndrome de Cushing iatrogénico.

Vol. 62(1): 5 - 15, 2021 
El tamaño de la muestra, fue de 90 niños, determinado por la fórmula: $\mathrm{n}=\mathrm{p} \times \mathrm{q} \times Z^{2} / \mathrm{P}^{2}$

$\mathrm{n}=$ tamaño de la muestra.

$Z=1,96$ constante. Expresa el nivel de confianza.

$\mathrm{p}=$ porcentaje de la población que tiene la característica de interés.

$\mathrm{q}=$ porcentaje de la población que no tiene la característica de interés.

$\mathrm{P}=$ precisión: error máximo permitido entre parámetro y estadístico, expresado en proporción.

A esta muestra se le realizó:

- Evaluación socioeconómica: Graffar Méndez-Castellano (16).

- Diagnóstico nutricional antropométrico: las mediciones fueron realizadas, de acuerdo a las normas y procedimientos internacionales. Para el peso y la talla se utilizó una balanza Detecto, las circunferencias se midieron con una cinta metálica flexible y los pliegues con un calibrador Lange. Se estudiaron:

1. IMC: obesidad > p97 (14).

2. Área grasa (AG) del brazo. Se utilizó como punto de referencia las tablas del Proyecto Venezuela, 1994 (17). - Reserva calórica normal: > p 10 a $\leq$ p 90 .

- Reserva calórica alta (sospecha de obesidad): > p90 a $\leq$ a p 97.

- Reserva calórica muy alta (obesidad): $>$ p 97.

3. Circunferencia de la cintura para la edad (CC). Se midió en la mitad de la distancia entre el borde inferior de la última costilla y el borde superior de la cresta ilíaca. Se consideró obesidad de tipo central, cuando el valor de la CC fue > p90 (18).

- Pruebas tiroideas. Se determinaron los valores de hormonas tiroideas (TSH, T3 libre y T4 libre) y se correlacionaron con sexo, condición socioeconómica, maduración sexual, variables antropométricas (IMC, AG del brazo y CG) y bioquímicas: insulina basal y Homeostasis Model Assesment (HOMA1-IR).

- Análisis de laboratorio. Después de 12 horas de ayuno, se extrajeron $10 \mathrm{~mL}$ de sangre de vena antecubital. Se realizaron las pruebas tiroideas TSH, T3 libre y T4 libre mediante el método ELISA de Monobind Inc., Lake Forest, USA, usando lector CDW100 Lavador/Incubador de Elisa automatizado, marca BIOLatin,Venezuela, y los resultados analizados de acuerdo a los valores de referencia del fabricante Monobind Inc. TSH: 0,35-5,5 UI/mL; T3 libre: 1,4-5 pg $/ \mathrm{mL}$ y T4 libre: 0,7-1,8 ng/dL (19).

La ǵlicemia se analizó por el método enzimático AA (línea liquida), de Wiener lab, BT-3000, Argentina, valor normal $<100$ $\mathrm{mg} / \mathrm{dL}$. La insulina se midió por el método Elisa de DRG, Springfield, USA, en un lector CDW-100 Lavador/Incubador de Elisa automatizado, marca BIOLatin. Se definió como hiperinsulinismo, a los niveles basales de insulinas $\geq$ a $15 \mu \mathrm{UI} / \mathrm{mL}$ (20). La sensibilidad insulínica basal, se calculó a través del índice HOMA1-IR $=$ insulina en ayuno $(\mu \mathrm{UI} / \mathrm{mL})$ $\mathrm{x}$ glicemia en ayuno $(\mathrm{mmol} / \mathrm{L}) / 22,5$. Considerándose resistencia a la insulina valores $>3,43$ (21).

- Análisis estadístico: Se realizaron cuadros de distribución de frecuencias con valores absolutos y porcentajes. Se comprobó la normalidad o no de la distribución de la muestra, usando la prueba de Kolmoronov Smirnov. Se estableció la tendencia central (media y mediana), las comparaciones de medias se analizaron a través de la "t de Student" y los valores de variables que no se adaptaron a la distribución normal, se contrastaron con la prueba no paramétrica de Mann- Whitney. Las correlaciones se evaluaron con el coeficiente de correlación de Spearman. La signnificancia estadística se estableció con un nivel del 5\% $(\mathrm{p}<0,05)$. Se empleó el paquete de análisis estadístico SPSS versión 19.

\section{RESULTADOS}

Los escolares, tenían un promedio de edad de 9,68 años, el 12,22\% $(n=11)$, con una condición económica de Graffar II, $52,22 \%(n=47)$ de Graffar III y 35,55\% (n= 32), con Graffar IV. 
El 50\% $(\mathrm{n}=45)$ del sexo masculino y $50 \%(n=45)$ del sexo femenino; $90 \%(n=81)$ con una maduración sexual Tanner I (Prepúber) y $10 \%(n=9)$ Tanner II (Púber). De los prepúberes, el 54,32\% $(\mathrm{n}=44)$ correspondió al sexo masculino y $45,68 \%(n=37)$, al femenino; mientras que en los púberes: $11,11 \%$ $(n=1)$ al sexo masculino y $88,88 \%(n=8)$ al femenino. Todos presentaron obesidad de tipo central $(\mathrm{CC} \geq \mathrm{p} 90)$ y un área grasa del brazo, con reserva calórica muy alta $(>\mathrm{p}$ 97).

En los estadísticos descriptivos de las variables en estudio, que se adaptaron a la distribución normal se observó en relación a la T4 libre, que los promedios estaban dentro de los valores normales, sin diferencia significativa en relación al sexo y que los promedios de AG del brazo y ǵlicemia basal fueron significativamente mayores $(\mathrm{p}<0,05)$ en el sexo masculino (Tabla I).

En la Tabla II, se muestran los valores de las variables bioquímicas que no se adaptaron a la distribución normal, sin diferencia significativa con relación al sexo; las medianas de la TSH y la T3 libre en la muestra total, se encontraron dentro de la normalidad.

En relación al funcionalismo tiroideo se observó que de los 90 escolares con obesidad, 3,33\% $(\mathrm{n}=3)$ presentó valores elevados de la hormona TSH, 10\% $(n=9)$ valores ba- jos de T3L y 12,22\% $(n=11)$ valores bajos de T4L; todas las pruebas sin diferencia significativa $(p>0,05)$, en relación al sexo (Tabla III).

El promedio de TSH normal fue de 2,00 $\mathrm{UI} / \mathrm{mL}$ y el elevado de $6,60 \mathrm{UI} / \mathrm{mL}$, con una diferencia estadísticamente significativa $(\mathrm{p}<0,05)$. El de T3 libre normal fue de 2,00 $\mathrm{pg} / \mathrm{mL}$ y el bajo $1,00 \mathrm{pg} / \mathrm{mL}$. El de T4 libre normal fue de $0,94 \mathrm{ng} / \mathrm{dL}$ y de T4 libre baja de $0,62 \mathrm{ng} / \mathrm{dL}$, con una diferencia estadísticamente significativa $(\mathrm{p}<0,05)$.

De los 90 pacientes, el 74,44\% $(n=67)$, fue eutiroideo y un $25,44 \%(n=23)$, presentó hipotiroidismo; de ellos, el 13,04\% $(n=3)$ tenía hipotiroidismo subclínico y el $86,95 \%$ $(n=20)$ hipotiroidismo hipotálamo-hipofisario o central.

No se encontró correlación $(\mathrm{p}>0,05)$ entre disfunción tiroidea y sexo, ni con estrato socioeconómico.

Al comparar las medias (Prueba t), de las variables antropométricas con el funcionalismo tiroideo, no se hallaron diferencias significativas $(\mathrm{p}>0,05)$. En relación a las variables bioquímicas, mediante la prueba de Mann-Whitney se encontraron valores de HOMA1-IR, significativamente más elevados en escolares con T4 libre baja $(\mathrm{p}<0,05)$, al compararlos con aquellos con T4 libre normal (Tabla IV).

TABLA I

VARIABLES ANTROPOMÉTRICAS Y BIOQUÍMICAS EN ESCOLARES CON OBESIDAD QUE SE ADAPTARON A LA DISTRIBUCIÓN NORMAL.

\begin{tabular}{lcccc}
\hline Variable & $\begin{array}{c}\text { Muestra total } \\
\mathrm{n}=90\end{array}$ & $\begin{array}{c}\text { Masculino } \\
\mathrm{n}=45\end{array}$ & $\begin{array}{c}\text { Femenino } \\
\mathrm{n}=45\end{array}$ & \\
\hline & $\mathrm{X} \pm \mathrm{DE}$ & $\mathrm{X} \pm \mathrm{DE}$ & $\mathrm{X} \pm \mathrm{DE}$ & $\mathrm{p}^{*}$ \\
Edad (años) & $9,68 \pm 1,35$ & $9,90 \pm 1,31$ & $9,45 \pm 1,36$ & $\mathrm{NS}$ \\
IMC $\left(\mathrm{Kg} / \mathrm{m}^{2}\right)$ & $25,10 \pm 3,30$ & $25,44 \pm 3,45$ & $24,75 \pm 3,16$ & $\mathrm{NS}$ \\
Área Grasa del brazo (AG) $\left(\mathrm{cm}^{2}\right)$ & $31,55 \pm 8,74$ & $33,66 \pm 8,71$ & $29,44 \pm 8,34$ & 0,02 \\
Circunferencia cintura (CC) $\mathrm{cm}$ & $81,24 \pm 8,06$ & $81,94 \pm 7,73$ & $80,54 \pm 8,41$ & $\mathrm{NS}$ \\
Glicemia basal $(\mathrm{mg} / \mathrm{dL})$ & $81,07 \pm 6,28$ & $83,26 \pm 5,09$ & $78,88 \pm 6,63$ & 0,001 \\
T4 libre (ng/dL) & $0,90 \pm 0,20$ & $0,90 \pm 0,26$ & $0,90 \pm 0,20$ & $\mathrm{NS}$ \\
\hline
\end{tabular}

"Prueba t de Student.

Vol. 62(1): 5 - 15, 2021 
TABLA II

VARIABLES BIOQUÍMICAS QUE NO SE ADAPTARON A LA DISTRIBUCIÓN NORMAL, EN ESCOLARES CON OBESIDAD.

\begin{tabular}{lccccccc}
\hline \multicolumn{1}{c}{ Variable } & \multicolumn{2}{c}{$\begin{array}{c}\text { Muestra total } \\
\mathrm{n}=90\end{array}$} & \multicolumn{2}{c}{$\begin{array}{c}\text { Masculino } \\
\mathrm{n}=45\end{array}$} & \multicolumn{2}{c}{$\begin{array}{c}\text { Femenino } \\
\mathrm{n}=45\end{array}$} \\
& $\mathrm{M}_{\mathrm{e}}$ & Rango & $\mathrm{M}_{\mathrm{e}}$ & Rango & $\mathrm{M}_{\mathrm{e}}$ & Rango & $\mathrm{p}^{*}$ \\
Insulina basal $(\mu \mathrm{UI} / \mathrm{mL})$ & 13,60 & 114,30 & 11,7 & 30,20 & 14,90 & 114,30 & $\mathrm{NS}$ \\
HOMA1-IR & 2,82 & 21,23 & 2,52 & 6,16 & 3,08 & 21,23 & $\mathrm{NS}$ \\
TSH $(\mathrm{UI} / \mathrm{mL})$ & 2,00 & 6,10 & 1,90 & 6,00 & 2,20 & 5,40 & $\mathrm{NS}$ \\
T3 libre $(\mathrm{pg} / \mathrm{mL})$ & 2,00 & 3,80 & 2,00 & 3,30 & 2,00 & 3,60 & $\mathrm{NS}$ \\
\hline
\end{tabular}

$\mathbf{M}_{\mathrm{e}}$ : Mediana.

"Prueba de Mann- Whitney.

TABLA III

PRUEBAS DE FUNCIÓN TIROIDEA EN ESCOLARES CON OBESIDAD.

\begin{tabular}{|c|c|c|c|c|c|c|c|c|c|}
\hline \multirow[t]{3}{*}{$\begin{array}{l}\text { Pruebas } \\
\text { Tiroideas }\end{array}$} & \multicolumn{4}{|c|}{$\begin{array}{c}\text { Masculino } \\
\mathrm{n}=45\end{array}$} & \multicolumn{5}{|c|}{$\begin{array}{c}\text { Femenino } \\
\mathrm{n}=45\end{array}$} \\
\hline & \multicolumn{2}{|c|}{ Normal } & \multicolumn{2}{|c|}{ Alto } & \multicolumn{2}{|c|}{ Normal } & \multicolumn{2}{|c|}{ Alto } & \\
\hline & $\mathrm{n}$ & $\%$ & $\mathrm{n}$ & $\%$ & $\mathrm{n}$ & $\%$ & $\mathrm{n}$ & $\%$ & $\mathrm{p}^{*}$ \\
\hline \multirow[t]{3}{*}{$\mathrm{TSH}(\mathrm{UI} / \mathrm{mL})$} & 44 & 48,88 & 1 & 1,11 & 43 & 47,77 & 2 & 2,22 & NS \\
\hline & \multicolumn{2}{|c|}{ Normal } & \multicolumn{2}{|c|}{ Baja } & \multicolumn{2}{|c|}{ Normal } & \multicolumn{2}{|c|}{ Baja } & \\
\hline & $\mathrm{n}$ & $\%$ & $\mathrm{n}$ & $\%$ & $\mathrm{n}$ & $\%$ & $\mathrm{n}$ & $\%$ & $\mathrm{p}^{*}$ \\
\hline T3 libre (pg/mL) & 40 & 44,44 & 5 & 5,55 & 41 & 45,55 & 4 & 4,44 & NS \\
\hline T4 libre (ng/dL) & 38 & 42,22 & 7 & 7,77 & 41 & 45,55 & 4 & 4,44 & NS \\
\hline
\end{tabular}

"Correlación de Spearman.

TSH alta: $>5,5 \mathrm{UI} / \mathrm{mL} \quad$ T3 libre baja: $<1,4 \mathrm{pg} / \mathrm{mL} \quad$ T4 libre baja: $<0,7 \mathrm{ng} / \mathrm{dL}$

TABLA IV

COMPARACIÓN DEL FUNCIONALISMO TIROIDEO CON VARIABLES ANTROPOMÉTRICAS Y BIOQUÍMICAS.

\begin{tabular}{lcccccc} 
& \multicolumn{2}{c}{$\begin{array}{c}\text { TSH normal } \\
\text { y elevada }(\mathrm{UI} / \mathrm{mL})\end{array}$} & \multicolumn{2}{c}{$\begin{array}{c}\text { T4 libre normal } \\
\mathrm{y} \text { baja(ng/dL) }\end{array}$} & \multicolumn{2}{c}{$\begin{array}{c}\text { T3 libre normal y baja } \\
(\mathrm{pg} / \mathrm{mL})\end{array}$} \\
\hline Variables Antropométricas & $\mathrm{t}$ & $\mathrm{p}$ & $\mathrm{t}$ & $\mathrm{p}$ & $\mathrm{t}$ & $\mathrm{p}$ \\
\hline IMC $\left(\mathrm{Kg} / \mathrm{m}^{2}\right)$ & $-0,21$ & $\mathrm{NS}$ & $-1,38$ & $\mathrm{NS}$ & $-0,86$ & $\mathrm{NS}$ \\
$\mathrm{CG}(\mathrm{cm})$ & 0,37 & $\mathrm{NS}$ & $-1,47$ & $\mathrm{NS}$ & $-0,73$ & $\mathrm{NS}$ \\
AG del brazo $\left(\mathrm{cm}^{2}\right)$ & $-0,17$ & $\mathrm{NS}$ & $-1,38$ & $\mathrm{NS}$ & $-1,14$ & $\mathrm{NS}$ \\
& & & & & & \\
\hline Variables Bioquímicas & $\mathrm{Z}$ & $\mathrm{p}$ & $\mathrm{Z}$ & $\mathrm{p}$ & $\mathrm{Z}$ & $\mathrm{p}$ \\
\hline HOMA1-IR & $-0,68$ & $\mathrm{NS}$ & $-2,27$ & $0,02^{*}$ & $-0,68$ & $\mathrm{NS}$ \\
Insulina basal $(\mu \mathrm{UI} / \mathrm{mL})$ & $-0,66$ & $\mathrm{NS}$ & $-1,78$ & $\mathrm{NS}$ & $-0,66$ & $\mathrm{NS}$ \\
\hline t: Prueba t de Student. Z: Prueba de Mann-Whitney. & $* \mathrm{p}<0,05$. & & &
\end{tabular}


En los escolares con obesidad y disfunción tiroidea, la T4 libre baja se correlacionó con hiperinsulinismo $(\mathrm{p}=0,02)$, pero no con resistencia a la insulina $(\mathrm{p}=0,10)$, (Tabla V).

De los 23 escolares con disfunción tiroidea, el 91,30\% $(n=21)$ fue prepúberes y un 8,69\% (n=2) púberes. En los prepúberes la disfunción tiroidea fue mayor en el sexo masculino $(n=13)$, que en el femenino $(n=8)$ y en púberes solamente se evidenció en el sexo femenino $(n=2)$, (Tabla VI).

No se encontraron diferencias significativas $(p>0,05)$, al comparar valores de TSH, T3 y T4 libre entre prepúberes y púberes. Los tres escolares con TSH elevada, con un promedio de 6,60 UI/mL, eran prepúberes. En relación a los 9 escolares con la T3 libre baja, 7 fueron prepúberes y 2 púberes, ambos grupos con un promedio de la hormona de 1,00 $\mathrm{pg} / \mathrm{mL}$. Los que tuvieron T4 libre baja, con un promedio de $0,62 \mathrm{ng} / \mathrm{dL}$, eran prepúberes.

\section{DISCUSIÓN}

La obesidad, especialmente la obesidad central, está relacionada con muchas anor- malidades endocrinas, incluida la disfunción tiroidea (22). En este estudio, donde un alto porcentaje de los pacientes pertenecía a familias de los estratos sociales de clase media baja y pobreza relativa, se encontró que la cuarta parte de los escolares con obesidad, mostró hipotiroidismo y el resto tenía una función normal, lo que representó una cantidad elevada en esta muestra.

Las posibles causas de los casos de hipotiroidismo subclínico con TSH elevada y T3 y T4 libres en concentraciones normales, incluyen disfunción neuroendocrina, resistencia a las hormonas tiroideas, modificación de la producción de TSH mediada por la leptina, y mutaciones del receptor de TSH (23). Nuestros resultados respecto al hipotiroidismo subclínico, concuerdan con estudios internacionales, donde se estima una prevalencia en niños obesos entre 7,5 y $23 \%$ $(6,9,24-26)$. La obesidad tiene influencia sobre la actividad del eje hipotálamo - hipófisis - tiroides, lo cual se evidencia mediante una elevación de la TSH en ausencia de déficit de Iodo y de alguna enfermedad tiroidea autoinmune $(3,6)$. Los niveles de TSH dis-

TABLA V

RELACIÓN ENTRE DISFUNCIÓN TIROIDEA, INSULINA BASAL Y RESISTENCIA A LA INSULINA (HOMA1-IR), EN ESCOLARES CON OBESIDAD.

\begin{tabular}{lccccc}
\hline & \multicolumn{1}{c}{ Insulina basal normal $(\mu \mathrm{UI} / \mathrm{mL})$} & Hiperinsulinismo $(\mu \mathrm{UI} / \mathrm{mL})$ & \\
\hline Pruebas Tiroideas & $\mathrm{n}$ & $\%$ & $\mathrm{n}$ & $\%$ & Total \\
\hline TSH Elevada $(\mathrm{UI} / \mathrm{mL})$ & 2 & 66,66 & 1 & 33,33 & 3 \\
T3 libre Baja (pg/mL) & 5 & 55,55 & 4 & 44,44 & 9 \\
T4 libre Baja (ng/dL) & 3 & 27,27 & 8 & $72,73^{*}$ & 11
\end{tabular}

HOMA1-IR Normal

RI

\begin{tabular}{lccccc}
\hline Pruebas Tiroideas & $\mathrm{n}$ & $\%$ & $\mathrm{n}$ & $\%$ & Total \\
\hline TSH Elevada $(\mathrm{UI} / \mathrm{mL})$ & 3 & 100 & 0 & 0 & 3 \\
T3 libre Baja (pg/mL) & 5 & 55,55 & 4 & 44,44 & 9 \\
T4 libre Baja (ng/dL) & 4 & 36,36 & 7 & $63,64^{*}$ & 11 \\
\hline
\end{tabular}

* Correlación de Spearman: Hiperinsulinismo p = 0,02

RI: Resistencia a la insulina $\mathrm{p}=0,10$

Vol. 62(1): 5 - 15, 2021 
TABLA VI

RELACIÓN ENTRE DISFUNCIÓN TIROIDEA Y MADURACIÓN SEXUAL.

\begin{tabular}{lccccccccc}
\hline & \multicolumn{3}{c}{ Prepúber $\mathrm{n}=81$} & \multicolumn{7}{c}{ Púber $\mathrm{n}=9$} \\
\hline & \multicolumn{1}{c}{ Masculino } & \multicolumn{2}{c}{ Femenino } & \multicolumn{2}{c}{ Masculino } & \multicolumn{2}{c}{ Femenino } \\
\hline Pruebas Tiroideas & $\mathrm{n}$ & $\%$ & $\mathrm{n}$ & $\%$ & $\mathrm{n}$ & $\%$ & $\mathrm{n}$ & $\%$ & Total \\
TSH Normal & 43 & 47,77 & 35 & 38,88 & 1 & 1,11 & 8 & 8,88 & 87 \\
TSH Elevada & 1 & 1,11 & 2 & 2,22 & 0 & 0 & 0 & 0 & 3 \\
\hline Total & 44 & 48,88 & 37 & 41,11 & 1 & 1,11 & 8 & 8,88 & 90 \\
\hline & & & & & & & & & \\
T3 libre Normal & 39 & 43,33 & 35 & 38,88 & 1 & 1,11 & 6 & 6,66 & 81 \\
T3 libre Baja & 5 & 5,55 & 2 & 2,22 & 0 & 0 & 2 & 2,22 & 9 \\
\hline Total & 44 & 48,88 & 37 & 41,11 & 1 & 1,11 & 8 & 8,88 & 90 \\
\hline & & & & & & & & & \\
T4 libre Normal & 37 & 41,11 & 33 & 36,66 & 1 & 1,11 & 8 & 8,88 & 79 \\
T4 libre Baja & 7 & 7,77 & 4 & 4,44 & 0 & 0 & 0 & 0 & 11 \\
\hline Total & 44 & 48,88 & 37 & 41,11 & 1 & 1,11 & 8 & 8,88 & 90 \\
\hline
\end{tabular}

minuyen cuando las personas obesas pierden peso, por tanto la elevación de TSH parece ser el resultado de la obesidad más que la causa (7). La teoría adaptativa establece que las hormonas tiroideas aumentan para incrementar el gasto energético y así disminuir la obesidad, este proceso podría estar mediado por la leptina, ya que se ha visto que los obesos tienen niveles más elevados de esta hormona, la cual podría estimular directamente la secreción de TSH (3). La literatura refiere, que la leptina podría estimular la conversión de T4 a T3, lo que podría ser interpretado como un mecanismo para favorecer la pérdida de peso, ya que la disminución de T4 estimularía la producción de TSH (6).

De los obesos con hipotiroidismo, un alto porcentaje presentó T3 libre baja, o T4 libre baja, con TSH normales, es decir no hubo una respuesta compensadora de la glándula tiroides para normalizar los valores de las hormonas periféricas, por falta de estímulo hipotálamo o hipofisiario (13). Estos resultados difieren de estudios internacionales, quienes hallaron mayormente hipotiroidismo del tipo subclínico $(6,9,24-26)$. El hipotiroidismo primario constituye la enfer- medad endocrinológica más frecuente de la infancia, las formas hipotálamo-hipofisarias son poco frecuentes y generalmente se asocian a otras deficiencias hormonales, que son las que destacan y conducen al diagnóstico. La resistencia periférica a las hormonas tiroideas es todavía menos frecuente $(3,12)$. La enfermedad no tiroidea puede dar lugar también a este patrón de secreción hormonal en el que típicamente, la TSH está en rango normal y la T3 libre baja (síndrome de T3 baja), pero esta situación es frecuente en niños recién nacidos prematuros, y está relacionada con la malnutrición y mayor morbilidad (13). Quizás los resultados de este estudio, se asocian a otras deficiencias hormonales y probablemente reflejan el hecho de que los pacientes son examinados en diferentes momentos, pudiendo también influir una alimentación excesiva o el ayuno, los cuales se asocian a una disminución de hormonas tiroideas.

En analogía con el estudio de Jin (24), no se encontró correlación de la TSH en suero con resistencia a la insulina, en contraste con otros reportes $(27,28)$. Los escolares con obesidad y T4 libre baja se correlaciona- 
ron significativamente con hiperinsulinismo $\mathrm{y}$ presentaron promedios mayores de HOMA1-IR. El aumento de los niveles de TSH y la disminución de los niveles de T4 libre se han asociado con marcadores elevados de resistencia a la insulina (29). Las hormonas tiroideas tienen un efecto significativo sobre el metabolismo de la glucosa y el desarrollo de resistencia a la insulina. En el hipotiroidismo los datos disponibles sugieren que prevalece la resistencia a la insulina de los tejidos periféricos $(6,30)$.

A semejanza con Thiagarajan y col (31), no se encontró correlación de las pruebas tiroideas con las variables antropométricas en estudio. Estos hallazgos difieren a lo reportado por la literatura $(9,24,26,32)$, donde señalan que los niveles séricos de TSH aumentan en paralelo con el IMC, la CG y la adiposidad, existiendo una correlación negativa entre los valores de T4 libre y el IMC. Estas diferencias pudieran deberse, a que por las escasas publicaciones de disfunción tiroidea en la edad escolar, se realizaron comparaciones con estudios que incluyeron preescolares y adolescentes; es conocido que la composición corporal en relación a masa magra y adiposidad varía según el grupo etario (33).

Los valores de hormonas tiroideas no presentaron diferencias, en relación con el grado de maduración sexual y sexo, al igual como lo han señalado algunos autores ( 25 , 27,32). Todos los escolares con TSH elevada eran prepúberes. Algunos autores consideran que en condiciones normales, estos niños presentan una hipertirotropinemia fisiológica y transitoria, debido a que es reversible al alcanzar el desarrollo puberal (9). Se ha descrito que sería la edad más que el estado puberal, lo que explicaría los niveles elevados de TSH encontrados en edades tempranas. En niños, el eje hipotálamo-hipófisis-tiroides va progresivamente madurando, desde la gestación hasta completar la pubertad (34).

A pesar de las limitaciones del estudio, como fueron el pequeño tamaño de la muestra, falta de valores de referencia de pruebas tiroideas propios de la población y no tener un grupo control de escolares eutróficos, razones por las cuales no se pueden establecer conclusiones definitivas, esta investigación es un modelo representativo de escolares con obesidad, cuyo procesamiento de hormonas tiroideas se realizó con una misma técnica y equipo. En conclusión, se encontró una frecuencia elevada de hipotiroidismo en escolares con obesidad, comparable a la descrita a nivel internacional. Aunque existe discrepancia en la interpretación y en los niveles de las hormonas tiroideas en los niños con obesidad, se justifica su tamizaje en la evaluación de estos pacientes.

\section{REFERENCIAS}

1. Chacín M, Carrillo S, Rodríguez J, Salazar J, Rojas J, Añez R, Angarita L, Chaparro Y, Martínez M, Anderson H, Reyna N, Herazo Y, Bermúdez V. Obesidad infantil: un problema de pequeños que se está volviendo grande. Rev Latinoam de Hipertensión [En línea] 2019 [Citado 2020 Jul 15]. Disponible en: https:// revhipertension.com/ rlh_5_2019/16_obesidad_infantil_problema.pdf

2. UNICEF-OMS-Banco Mundial: Estimaciones conjuntas de desnutrición infantil. $\mathrm{Ni}$ veles y tendencias. [En línea] 2019 [Citado 2020 Jul 15]. Disponible en: https://www. who.int/nutgrowthdb/estimates/en/

3. Álvarez P, Sangiao S, Brandón I, Cordido F. Función endocrina en la obesidad. Endocrinol Nutr 2011;58(8):422-432.

4. Dayal D, Kumar T. Highh prevalence of thyroid dysfunction in children with simple obesity. Indian J Pediatr 2019;56(4):331.

5. Sanyal D, Raychaudhuri M. Hypothyroidism and obesity: An intriguing link. Indian J Endocrinol Metab 2016; 20 (4): 554-557.

6. Reinehr T. Obesity and thyroid function. Mol Cell Endocrinol 2010; 316: 165-171.

7. Pacifico L, Anania C, Ferraro F, Andreoli G, Chiesa C. Thyroid function in childhood obesity and metabolic comorbidity. Clin Chim Acta 2012; 413: 396-405.

8. Kratzsch J, Schubert G, Pulzer F, Pfaeffle R, Koerner A, Dietz A, Rauh M, Kiess W, Thiery J. Reference intervals for TSH and thyroid hormones are mainly affected by

Vol. 62(1): 5 - 15, 2021 
age, body mass index and number of blood leucocytes, but hardly by gender and thyroid autoantibodies during the first decades of life. Clin Biochem 2008; 41: 1091-1098.

9. Ayala M, Guerrero J, Vergara A, Salazar G, Cruz D. Función tiroidea en población pediátrica con diferente estado nutricional. Bol Med Hosp Infant Mex 2018;75(5):279-286.

10. Patel R, Dave C, Mehta S, Mendpara H, Shukla R, Bajpai A. Metabolic Impact of subclinical hypothyroidism in obese children and adolescents. Indian J Pediatr [En línea] 2020 [Citado 2020 Agost 25] Disponible en: https://pubmed.ncbi.nlm.nih. gov/32797391/

11. Guerra K. Prevalencia de hipotiroidismo subclínico. Consulta de Endocrinología Pediátrica. Hospital de niños "Dr. Jorge Lizarraga". [Tesis Especialista] Valencia: Univ de Carabobo: 2017. [En línea] 2017 [Citado 2020 Agost 25] Disponible en: http://mriuc.bc.uc.edu.ve/ bitstream/handle/123456789/7601/kguerra.pdf? sequence $=1$

12. Muñoz J. Árboles de decisión en patología tiroidea. SEMG Catalunya [En línea] 2016 [Citado 2020 Abr 5]. Disponible en: http:// www.semg.es/granada2016/imaǵes/stories/recursos/doc_complementaria/Archivo-para-descargar-participantes-en-TallerManejo-Patologia-Tiroidea.pdf

13. Mayayo E, Ferrández A, Labarta J. Interpretación de las pruebas tiroideas. An Esp Pediatr 2002;56(S 4):42-52.

14. World Health Organization. Growth reference 5-19 years [En línea] 2007 [Citado 2020 Abr 6]. Disponible en: https://www. who.int/growthref/en/

15. Izaguirre de Espinoza I, López M. Evaluación del crecimiento y la maduración física. Nutrición Pediátrica. 1a Ed. Caracas (Vzla): Editorial Médica Panamericana; 2009, p 10.

16. Méndez H, Méndez M. Sociedad y estratificación. Método Graffar-Méndez Castellano. Fundacredesa 1994.

17. Landaeta M. Área Grasa. Fundacredesa. Proyecto Venezuela. Arch Venez Puer Ped 1998; 61:supl 1.

18. Me Carthy H, Jarret K, Crawley H. The development of waist circumference percentiles in British children aged 5 to 16.9. Eur J Clin Nutr 2001;55:902-907.
19. Cooper D, Krainik A, Lubner S, Reno H. Manual Washington de Terapéutica Médica. 32a Ed. Washington (EEUU): Spectracef: 2007.

20. Ten S, Maclaren N. Insulin resistance syndrome in children. J Clin Endocrinol Metab 2004; 89:2526-2539.

21. García B, García C, Jiménez C, González A, Calvo C, Alcázar M, Díaz E. Índice HOMA y QUICKI, insulina y péptido C en niños sanos. Puntos de corte de riesgó cardiovascular. An Pediatr (Bare) 2007; 66(5):481-490.

22. An YM, Moon S, Kim S, Suh Y, Lee J. Thyroid function in obese Korean children and adolescents: Korea National Health and Nutrition Examination Survey 2013-2015. Ann Pediatr Endocrinol Metab 2018;23(3):141-147.

23. Ibáñez L, Mareos M. Actualización en patología tiroidea. En: AEPap (ed). Curso de Actualización Pediatría. Madrid: Lúa Ediciones; 2017. P 161-174.

24. Jin H. Prevalence of subclinical hypothyroidism in obese children or adolescents and association between thyroid hormone and the components of metabolic syndrome. $J$ Paediatr Child Health 2018;54(9):975-980.

25. Sánchez B, Godoy S, García B, Barja S. Niveles de hormonas tiroideas en niños obesos. Rev Chil Pediatr 2014; 85 (3): 288-297.

26. Habib A, Molayemat M, Habib A. Elevated serum TSH concentrations are associated with higher BMI $Z$-scores in southern Iranian children and adolescents. Thyroid Res 2020;13:9.

27. Kara O. Influence of subclinical hypothyroidism on metabolic parameters in obese children and adolescents. Clin Exp Pediatr 2020;63(3):110-114.

28. Longhi S, Radetti G. Thyroid function and obesity. J Clin Res Pediatr Endocrinol 2013;5:40-44.

29. Hainer V, Zamrazilová H, Aldhoon H. Are the thyroid hormones and thyrotropin associated with cardiometabolic risks and insulin resistance even in euthyroid subjects? Vnitr Lek 2016;62(S 3):63-67.

30. Gierach M, Gierach J, Junik R. Insulin resistance and thyroid disorders. Endokrynol Pol 2014;65(1):70-76. 
31. Thiagarajan S, Arun B, Balaji R. Subclinical hypothyroidism in obese south Indian children. Indian J Pediatr [En línea] 2019; 86:662 [Citado 2020 Agost 15] Disponible en: https://doi.org/10.1007/s12098-01902966-9.

32. Dahl M, Dam J, Esmann C, Tonsgaard J, Pedersen O, Hansen T. Subclinical hypothyroidism in Danish thin and obese children and adolescents .J Clin Res Pediatr Endocrinol 2017; 9(1):8-16.
33. Cordero R, Zabala M, Infante R, Hagel I. Composición corporal y el patrón de grasa en niños y niñas en edad escolar de zonas rurales y urbanas de Venezuela. Tribuna del Investig 2014;15(1-2):46-55.

34. Önsesveren I, Barjaktarovic M, Chaker L, Rijke Y, Jaddoe V, Van Santen H, Visser T, Peeters R, Korevaar T. Childhood thyroid function reference ranges and determinants: A Literature overview and a prospective cohort study. Thyroid 2017;27(11):1360-1369. 